Urologe $2020 \cdot 59: 487$

https://doi.org/10.1007/s00120-020-01171-3

Online publiziert: 11. März 2020

(c) Springer Medizin Verlag $\mathrm{GmbH}$, ein Teil von Springer Nature 2020
Marc-Oliver Grimm ${ }^{1}$ Christian Doehn ${ }^{2}$ - Susanne Krege ${ }^{3}$

${ }^{1}$ Klinik und Poliklinik für Urologie, Universitätsklinikum Jena, Jena, Deutschland

${ }^{2}$ Urologikum Lübeck, Lübeck, Deutschland

${ }^{3}$ Klinik für Urologie, Kinderurologie und urologische Onkologie, Kliniken Essen-Mitte, Evang. Huyssens Stiftung/Knappschaft GmbH, Essen, Deutschland

\section{Erratum zu: Nierenzellkarzinom}

\section{Erratum zu:}

Urologe 2020

https://doi.org/10.1007/s00120-020-

01130-y

Leider wurde durch einen Fehler im Lektorat die Korrespondenzadresse und die zugehörigen Affiliationen von Herrn Prof. Dr. Doehn, in der „Einführung zum Thema" fehlerhaft dargestellt. Wir bitten die korrekten Informationen zu beachten und den Fehler zu entschuldigen.

\section{Korrespondenzadresse}

\section{Prof. Dr. Marc-Oliver Grimm}

Klinik und Poliklinik für Urologie, Universitätsklinikum Jena

Lessingstraße 1, 07743 Jena, Deutschland

Marc-Oliver.Grimm@med.uni-jena.de

\section{Prof. Dr. Christian Doehn}

Urologikum Lübeck

Am Kaufhof 2, 23566 Lübeck, Deutschland

doehn@urologikum-luebeck.de

\section{Prof. Dr. Susanne Krege}

Klinik für Urologie, Kinderurologie und urologische Onkologie, Kliniken Essen-Mitte, Evang. Huyssens Stiftung/Knappschaft GmbH Henricistr. 92, 45136 Essen, Deutschland S.Krege@kem-med.com 